\title{
The Role of Intra-abdominal Pressure Measurement in Awake Rat Cystometry
}

\author{
Tack Lee, Sang-Min Yoon \\ Department of Urology, Inha University College of Medicine, Incheon, Korea
}

\begin{abstract}
Rat cystometry is increasingly being used in research on overactive bladder and is a research tool for investigating bladder functions during the storage and voiding phases. Measurement of the pressure in the bladder is an essential part of cystometry, although that pressure is the sum of both detrusor and intra-abdominal pressures. In anesthetic cystometry, measurement of the intra-abdominal pressure is not necessary, because the values of this variable are negligible. In awake cystometry, however, it is mandatory to separate the changes in intra-abdominal pressure from those in intravesical pressure, because consciousness causes much variability in intra-abdominal pressure. This review describes why we use the term "detrusor overactivity" in animal research with intra-abdominal pressure and presents evidence for the role of intra-abdominal pressure in the cystometry of normal rats and in animal models of overactive bladder directed at understanding the pathogenesis of the overactive bladder condition. The methodology is also briefly reviewed.
\end{abstract}

Keywords: Urinary bladder; Overactive; Physiology; Rat; Urodynamics

\section{INTRODUCTION}

The lower urinary tract including the bladder and urethra forms a functional unit that is governed by complicated and only partially disclosed interactions between the central and peripheral nervous systems that require further exploration or clarification $[1,2]$. Cystometry is a diagnostic procedure used to assess the functions of lower urinary tract by measuring the pressures and volumes related to the bladder. However, the pressures measured in the bladder, which is labeled as intravesical pressure (IVP), contains the detrusor pressure (DP), which we want to know, mixed with the intra-abdominal pressure (IAP) [3]. In order to measure the DP in human cystometry, we routinely use subtraction cystometry, in which the DP is shown by subtracting the IAP from measured IVP, which is done automatically by the urodynamic machine in real time. This is not the case in animal cystometry. Our group first reported a technique for measuring
IAP in rat cystometry in 2008 [4]. We realized the importance of IAP in discrimination between detrusor overactivity and abdominal straining. Those two different activities have a similar appearance when only the IVP curve is analyzed but can be discriminated by the analysis of IAP (Fig. 1) [5,6].

In the present article, the role of IAP in the cystometry of normal and diseased rat models is briefly reviewed, with a brief explanation of the methodology.

\section{METHODOLOGY FOR IAP IN RAT CYSTOMETRY}

\section{Rationale for the Use of Balloon}

In human cystometry, the use of a balloon catheter in the rectum or vaginal cavity is recommended for the measurement of IAP [3]. The rationale for the use of a balloon is to prevent the intrusion or blockage of the fecal materials or vaginal wall into
Corresponding author: Tack Lee

Department of Urology, Inha University Hospital, Inha University College of Medicine, 27 Inhang-ro, Jung-gu, Incheon 400-711, Korea

Tel: +82-32-890-3448 / Fax: +82-32-890-3097 / E-mail: lee.tack33@gmail.com Submitted: May 20, 2013 / Accepted after revision: June 18, 2013
This is an Open Access article distributed under the terms of the Creative Commons Attribution Non-Commercial License (http://creativecommons.org/licenses/by-nc/3.0/) which permits unrestricted non-commercial use, distribution, and reproduction in any medium, provided the original work is properly cited. 

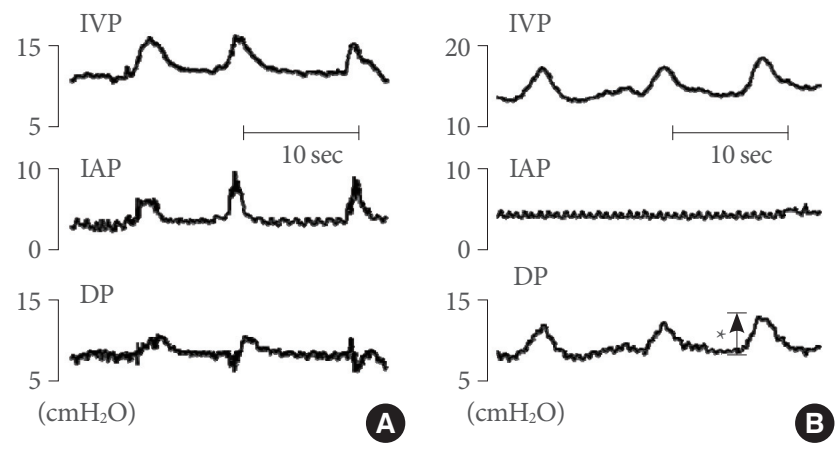

Fig. 1. Cystometrogram showing (A) abdominal straining and (B) detrusor overactivity with intravesical pressures (IVPs) and intra-abdominal pressures (IAPs). (A) The abdominal straining, which shows IVP rises with simultaneous changes in IAP. (B) The detrusor overactivity, which shows IVP rises without simultaneous changes in IAP. DP, detrusor pressure. ${ }^{*}$ Increased amplitude of detrusor overactivity. Adapted from Jin LH, et al. Scand J Urol Nephrol 2009;43:3-7 [5].

the opening of the catheter tip and to make it possible to transmit the pressure to the transducer continuously by maintaining a small volume of fluid at the tip of the catheter. To record IAP in rat cystometry, we used a $0.05 \mathrm{~mL}$ balloon (Latex, Daewoo Medical, Incheon, Korea) on the tip cuff of a PE-50 polyethylene catheter, to prevent the intrusion of the omentum or bowel, placed in the intra-abdominal space [4]. Overfilling or distention of the balloon will result in an overexpressed response of pressure changes, similar to human cystometry. The balloon works best when it is stabilized between 80 and $100 \mathrm{cmH}_{2} \mathrm{O}$. A common mistake in the measurement of IAP in rat cystometry is leakage through the multiple joints of the lines and connectors. If the IAP drops spontaneously in the resting condition, linkage may be indicated, and Parafilm should be wrapped around the connectors of the multiple lines $[7,8]$.

\section{The Position of the Balloon}

The bladder is located inside the body wall that is composed of a number of muscles. Thus, the pressure changes recorded in the bladder represent those caused by the contractions of the bladder and of the outside muscles. The goal of cystometry is to assess detrusor activity [3], for which the contractions of outside muscles, that is, the perivesical pressures, must be excluded. These pressures surrounding the bladder suggests the sum of pressures delivered from all directions to the bladder - anterior, posterior, superior, right lateral, left lateral, and sometimes from below [4]. Because it is impossible to check the true sum from these six directions, we located the balloon proximal to the bladder, which is the initial point on the line that urine travels during voiding. The balloon should not be in direct contact with the bladder, which would result in the IAP being erroneously affected by the filled bladder. Thus, we attached the balloon with a silk tie to the bladder-pressure recording catheter at a fixed distance of $1 \mathrm{~cm}$ to the bladder. If the distance is too long, the pressures would not be representative.

\section{Set Zero or Balance of IAP}

In human cystometry, the real pressures that are recorded directly from the body are IVP and IAP. The DP is the value automatically calculated from the IVP and IAP in the real-time process. Therefore, for this direct comparison of the different pressures, a process of zeroing to reference height is needed. Zero pressure is the value recorded when a transducer is open to the environment, and the reference height is set as the upper edge of the symphysis pubis in humans [3]. In our method in animals, the zeroing to the reference height is set to the level of the rat's bladder for both pressures, but the IAP requires one more step after the cystometry, because the balloon pressure is high $[4,8]$. IAP is defined as the recorded balloon pressure corrected by subtracting the lowest balloon pressure in each voiding cycle, which is comparable to zeroing in human cystometry $[4,9]$.

\section{IAP IN NORMAL VOIDING}

Normal voiding is initiated and maintained by urethral relaxation followed by detrusor contraction, and abdominal straining is not always necessary. However, some humans, especially women, can initiate and maintain their micturition only by urethral relaxation and abdominal straining without detrusor contraction $[10,11]$. This suggests that the straining at voiding may have some other role in voiding that is not always pathologic. This can be initiated either voluntarily and consciously or instinctively and unconsciously and can result in pathologic or physiologic conditions. Patients with detrusor areflexia sometimes use pathologic straining, which leads to structural bladder wall changes such as trabeculations and diverticula [12]. However, recent animal studies have shown proof of physiologic straining in normal voiding. Cruz and Downie [11] investigated in healthy female rats whether the abdominal muscle contracts during voiding. They showed with electromyograms that the abdominal muscle is reflexively and consistently activated during physiological urine expulsion. In 2008, our group 


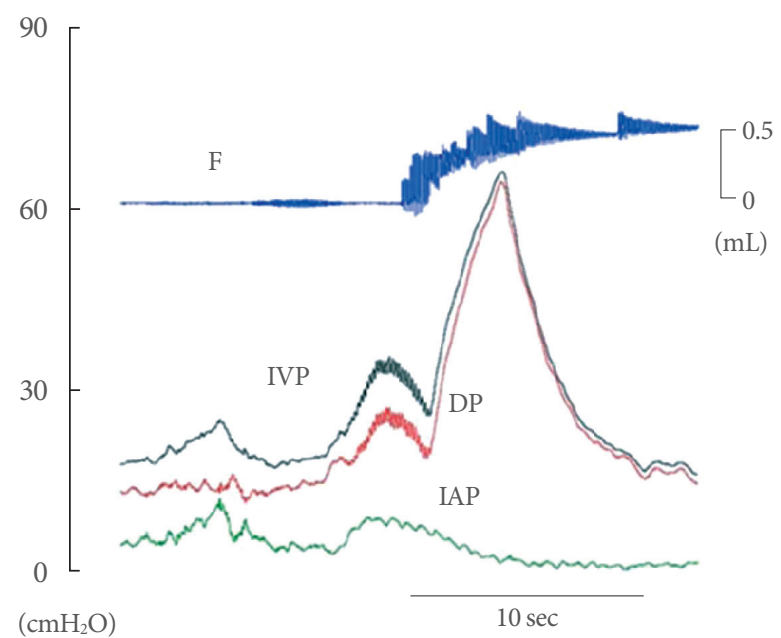

Fig. 2. Representative tracing showing the relations between intravesical, intra-abdominal, and detrusor pressure and flow of urine. IAP, intra-abdominal pressure; DP, detrusor pressure; IVP, intravesical pressure; F, flow; MP, micturition pressure. Adapted from Lee T, et al. Neurourol Urodyn 2008;27:88-95 [4].

proved using the IAP methodology that all conscious rats of both sexes use this physiologic abdominal straining (Fig. 2) [4]. From a physics viewpoint, the contracting power of the bladder alone is not enough to allow the urine go out efficiently into the urethra. Some additional driving force is needed to deliver this power in one direction such as into the urethra. This abdominal straining seems to be a physiologic action in voiding that is induced instinctively and unconsciously.

\section{IAP IN THE ANIMAL MODEL OF DISEASE}

\section{DP in Rat Cystometry}

The DP, which is indicative of detrusor activity, is the final goal of cystometry. In rat cystometry under anesthesia, measurement of the DP is not necessary, because the rats cannot strain their abdomen consciously and the DP is the same as IVP. In awake rat cystometry, however, the conscious contractions of many muscles in the body wall affect the IVP, and correction of the measurement by IAP is needed to get the DP. The IAPs are higher and more variable in the voiding phase than in the storage phase (Fig. 3) [4].

\section{Detrusor Overactivity and NVC}

Overactive bladder $(\mathrm{OAB})$ is characterized by the presence of urinary urgency, which usually corresponds to the presence of detrusor contractions during the storage phase in urodynamic

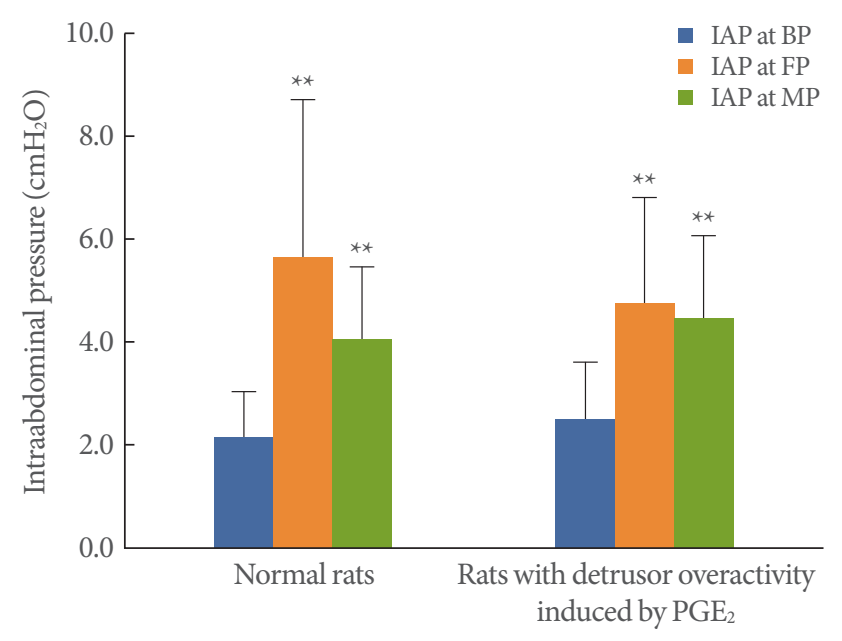

Fig. 3. Comparison of intra-abdominal pressure (IAP) as basal pressure (BP), flow pressure (FP), and micturition pressure (MP) in all rats of both sexes and with normal bladder function and detrusor overactivity induced by prostaglandin E2 (PGE2). All rats, of both sexes $(n=12)$, exhibited abdominal straining during voiding (recorded as increases in abdominal pressure), which significantly affected FP and MP compared with $\mathrm{BP} .{ }^{*} \mathrm{P}<0.01$ versus IAP at BP. Adapted from Lee T, et al. Neurourol Urodyn 2008;27:88-95 [4].

study $[13,14]$. The animal models of OAB show phasic contractions of the IVP curve during the storage phase, which have been reported as NVCs in rat cystometry, especially anesthetic cystometry $[15,16]$. This has been used as the core surrogate for urgency in basic research on OAB. However, there is still some confusion in the use of the terms NVC and detrusor overactivity. In human cystometry, the detrusor overactivity is the urodynamic term and is based on the IVP and IAP findings [3]. It is defined as an IVP change without a simultaneous change in IAP. Without an IAP finding, we do not call it detrusor overactivity. The NVC in animal cystometry is based on only IVP findings. Applying the IAP findings to the NVC characterizes the urodynamic diagnosis of detrusor overactivity.

In our method, we tried to quantify the detrusor overactivity as frequency per minute or increased amplitude from base to peak of the spike [5,6]. At first, we counted the IVP rises that exceeded $2 \mathrm{cmH}_{2} \mathrm{O}$ from baseline during the storage phase. With IAP findings, these are interpreted as detrusor overactivity or abdominal straining.

With this methodology, we could use more sophisticated analysis for detrusor overactivity. The spontaneous hypertensive rats (SHRs) do not show detrusor overactivity before a certain age, such as 12 weeks of age [8], and Wistar-Kyoto rats are 
not an appropriate control in studies of detrusor overactivity because they show detrusor overactivity of similar frequency and amplitude as found in SHRs [7]. Furthermore, the substantial detrusor overactivity represented up to $76 \%$ of total IVP rises, with the remainder caused by abdominal straining [5], which could be erroneously interpreted as detrusor overactivity if measured without IAP. In awake rats, variations in IAP may lead to false reports of detrusor overactivity.

\section{CONCLUSIONS}

There is some confusion concerning the definition of detrusor overactivity. This is a urodynamic term in human cystometry, defined by IVP and IAP findings only, not by clinical information. Thus, this same definition can be applied to animal cystometry. The NVC is the term defined by IVP only, and detrusor overactivity is the term defined by both IVP and IAP findings.

Detrusor overactivity is the key target for OAB research using rat cystometry. In particular, awake rat cystometry requires the measurement of IAP because of its variability. This more sophisticated analysis using quantification of detrusor overactivity will lead to deeper insight into basic research on OAB.

\section{CONFLICT OF INTEREST}

No potential conflict of interest relevant to this article was reported.

\section{REFERENCES}

1. Andersson KE, Wein AJ. Pharmacology of the lower urinary tract: basis for current and future treatments of urinary incontinence. Pharmacol Rev 2004;56:581-631.

2. Andersson KE. Advances in the pharmacological control of the bladder. Exp Physiol 1999;84:195-213.

3. Schäfer W, Abrams P, Liao L, Mattiasson A, Pesce F, Spangberg A, et al. Good urodynamic practices: uroflowmetry, filling cystometry, and pressure-flow studies. Neurourol Urodyn 2002;21:261-74.

4. Lee T, Andersson KE, Streng T, Hedlund P. Simultaneous registration of intraabdominal and intravesical pressures during cystometry in conscious rats: effects of bladder outlet obstruction and in- travesical PGE2. Neurourol Urodyn 2008;27:88-95.

5. Jin LH, Andersson KE, Kwon YH, Park CS, Yoon SM, Lee T. Substantial detrusor overactivity in conscious spontaneously hypertensive rats with hyperactive behaviour. Scand J Urol Nephrol 2009;43: 3-7.

6. Jin LH, Park CS, Shin HY, Yoon SM, Lee T. Dissimilar effects of tolterodine on detrusor overactivity in awake rats with chemical cystitis and partial bladder outlet obstruction. Int Neurourol J 2011; 15:120-6.

7. Jin LH, Andersson KE, Kwon YH, Yoon SM, Lee T. Selection of a control rat for conscious spontaneous hypertensive rats in studies of detrusor overactivity on the basis of measurement of intra-abdominal pressures. Neurourol Urodyn 2010;29:1338-43.

8. Jin LH, Lee HJ, Shin HY, Choi BH, Yoon SM, Park CS, et al. Development and changes with age of detrusor overactivity in spontaneous hypertensive rats as observed by simultaneous registrations of intravesical and intraabdominal pressures. Int Neurourol J 2011;15: 192-8.

9. Jin LH, Andersson KE, Han JU, Kwon YH, Park CS, Shin HY, et al. Persistent detrusor overactivity in rats after relief of partial urethral obstruction. Am J Physiol Regul Integr Comp Physiol 2011;301: R896-904.

10. Yang JM, Huang WC. Implications of abdominal straining in women with lower urinary tract symptoms. Urology 2002;60:428-33.

11. Cruz Y, Downie JW. Sexually dimorphic micturition in rats: relationship of perineal muscle activity to voiding pattern. Am J Physiol Regul Integr Comp Physiol 2005;289:R1307-18.

12. Tanaka C, Nishino N, Hashimoto T, Kitamura N, Yoshihara C, Saito N. Second messenger systems in brains of patients with Parkinson's or Huntington's disease. Adv Neurol 1993;60:175-80.

13. Andersson KE. Overactive bladder: pharmacological aspects. Scand J Urol Nephrol Suppl 2002;(210):72-81.

14. Garnett S, Abrams P. The natural history of the overactive bladder and detrusor overactivity. A review of the evidence regarding the long-term outcome of the overactive bladder. J Urol 2003;169:843-8.

15. Juszczak K, Ziomber A, Wyczolkowski M, Thor PJ. Urodynamic effects of the bladder C-fiber afferent activity modulation in chronic model of overactive bladder in rats. J Physiol Pharmacol 2009;60: 85-91.

16. Rajasekaran M, Wilkes N, Kuntz S, E Albo M. Rho-kinase inhibition suppresses bladder hyperactivity in spontaneously hypertensive rats. Neurourol Urodyn 2005;24:295-300. 